\title{
Amenable groups that act on the line
}

\author{
DAVE WitTE MORRIS
}

\begin{abstract}
Let $\Gamma$ be a finitely generated, amenable group. Using an idea of É Ghys, we prove that if $\Gamma$ has a nontrivial, orientation-preserving action on the real line, then $\Gamma$ has an infinite, cyclic quotient. (The converse is obvious.) This implies that if $\Gamma$ has a faithful action on the circle, then some finite-index subgroup of $\Gamma$ has the property that all of its nontrivial, finitely generated subgroups have infinite, cyclic quotients. It also means that every left-orderable, amenable group is locally indicable. This answers a question of P Linnell.
\end{abstract}

20F60; 06F15, 37C85, 37E05, 37E10, 43A07, 57S25

\section{Introduction}

Let $\Gamma$ be an abstract group (with the discrete topology). It is obvious that if $\Gamma$ has an infinite cyclic quotient, then $\Gamma$ has a nontrivial, orientation-preserving action on the real line $\mathbb{R}$. The converse is not true in general, even for finitely generated groups $[4$, Example 6.9.2, page 128]. In this note, we use an idea of É Ghys to prove that the converse does hold in the class of finitely generated, amenable groups.

Definition 0.1 [10, page 9 and Theorem 5.4(i,iii), page 45]

- A measure $\mu$ on a measure space $X$ is said to be a probability measure iff $\mu(X)=1$.

- A (discrete) group $\Gamma$ is amenable iff for every continuous action of $\Gamma$ on a compact, Hausdorff space $X$, there is a $\Gamma$-invariant probability measure on $X$.

Theorem A Let $\Gamma$ be a finitely generated, amenable group. Then $\Gamma$ has a nontrivial, orientation-preserving action on $\mathbb{R}$ if and only if $\Gamma$ has an infinite cyclic quotient.

It is well known that a countable group has a faithful, orientation-preserving action on $\mathbb{R}$ if and only if it is left orderable [3, Theorem 6.8]. (That is, there is a left-invariant order on $\Gamma$; in other words, there is a total order $\prec$ on $\Gamma$, such that, for all $\gamma, \lambda_{1}, \lambda_{2} \in \Gamma$, if $\lambda_{1} \prec \lambda_{2}$, then $\gamma \lambda_{1} \prec \gamma \lambda_{2}$.) Also, every subgroup of an amenable group is amenable [10, Proposition 13.3]. Hence, the nontrivial direction of Theorem A can be stated in the following purely algebraic form. 
Definition 0.2 [4, page 127] A group is locally indicable iff each of its nontrivial finitely generated subgroups has an infinite cyclic quotient.

Theorem B Every amenable left-orderable group is locally indicable.

\section{Remarks}

(1) The theorem answers a question of $P$ Linnell [6, page 134].

(2) Every locally indicable group (whether amenable or not) is left orderable (Burns and Hale [1], [4, Lemma 6.9.1]).

(3) Theorem B has previously been proved with "amenable" replaced by stronger hypotheses, such as polycyclic (Rhemtulla [11]), solvable-by-finite (Chiswell and Kropholler [2]), supramenable (Kropholler [5]), or elementary amenable (Linnell [6]). There are also interesting results that replace "amenable" with the assumption that $\Gamma$ has no nonabelian free semigroups (Longobardi, Maj and Rhemtulla [8]), or generalizations of this (Longobardi, Maj and Rhemtulla [9], Linnell [7]).

(4) P Linnell [7, Conjecture 1.1] has conjectured that the theorem is valid with "amenable" replaced by the weaker condition of not containing any nonabelian free subgroups.

Because the universal cover of the circle $S^{1}$ is $\mathbb{R}$, the following corollary is an easy consequence (cf [7, Section 5]).

Corollary $\mathbf{C}$ If $\Gamma$ is an amenable group, and $\Gamma$ has a faithful, orientation-preserving action on $S^{1}$, then $\Gamma$ has a normal subgroup $N$, such that $N$ is locally indicable, and $\Gamma / N$ is a finite cyclic group.

Proof of Theorem B Let $\Gamma$ be an amenable group that is left orderable. We wish to show that $\Gamma$ is locally indicable, so there is no harm in assuming that $\Gamma$ is nontrivial and finitely generated (hence, countable). Details of each of the following steps of the proof (and the necessary definitions) are presented in the indicated section below.

Section 1 Let $\mathcal{O}$ be the collection of all left-invariant orders on $\Gamma$. Étienne Ghys observed that $\mathcal{O}$ is a compact Hausdorff space, and that the action of $\Gamma$ on $\mathcal{O}$ by right translations is continuous.

Section 2 Since $\Gamma$ is amenable, there is a $\Gamma$-invariant probability measure $\mu$ on $\mathcal{O}$.

Section 3 The Poincaré Recurrence Theorem implies there is a point in $\mathcal{O}$ that is recurrent for every cyclic subgroup of $\Gamma$.

Algebraic 83 Geometric Topology, Volume 6 (2006) 
Section 4 Being recurrent for every cyclic subgroup is a stronger condition than being Conradian, and it is well known that any group admitting a Conradian left-invariant order is locally indicable.

Therefore, $\Gamma$ is locally indicable.

Acknowledgments I would like to thank Étienne Ghys for pointing out (several years ago) that the space of orderings of $\Gamma$ is compact, and for suggesting that it would be worthwhile to study the action of $\Gamma$ on this space. I also benefitted from conversations with Uri Bader that clarified and extended Ghys' observations, and from further discussions with Alex Furman and Tsachik Gelander. Peter Linnell provided helpful comments on a preliminary version of this manuscript.

I would also like to thank the Department of Mathematics at the University of Chicago for their hospitality while this research was being carried out. The work was partially supported by a grant from the National Science and Engineering Research Council of Canada.

\section{The space of left-invariant orders}

Fix a left-orderable group $\Gamma$. In this section, we present an idea of É Ghys (personal communication). The space of left-invariant orderings was topologized in a paper of A S Sikora [12], but the action of $\Gamma$ on this space does not seem to have appeared previously in the literature.

\section{Definition 1.1}

(1) Let $\mathcal{O}$ be the collection of all left-invariant orders on $\Gamma$. (Note that $\mathcal{O}$ is nonempty, because $\Gamma$ is left orderable.)

(2) (Ghys, Sikora [12, Definition 1.1]) Topologize $\mathcal{O}$ by declaring a set to be open iff it is a union of basic open sets of the form

$$
U_{\lambda_{1}, \lambda_{2}, \ldots, \lambda_{r}}=\left\{\prec \mid \lambda_{1} \prec \lambda_{2} \prec \cdots \prec \lambda_{r}\right\}
$$

for a sequence of distinct $\lambda_{1}, \lambda_{2}, \ldots, \lambda_{r} \in \Gamma$.

(3) (Ghys) Let $\Gamma$ act on $\mathcal{O}$ (on the right) by right translation:

$$
\lambda_{1} \prec_{\gamma} \lambda_{2} \Longleftrightarrow \lambda_{1} \gamma^{-1} \prec \lambda_{2} \gamma^{-1} \text {. }
$$

It is clear that if $\prec$ is a left-invariant order, then $\prec_{\gamma}$ is a left-invariant order, for every $\gamma \in \Gamma$. Also, we have $\prec_{\gamma_{1} \gamma_{2}}=\left(\prec_{\gamma_{1}}\right)_{\gamma_{2}}$, so this defines an action of $\Gamma$. 


\section{Lemma 1.2}

(1) (Ghys, Sikora) $\mathcal{O}$ is a compact, Hausdorff space.

(2) (Ghys) $\Gamma$ acts on $\mathcal{O}$ by homeomorphisms.

\section{Proof}

(1) [12, Theorem 1.4] The collection $\mathcal{P}(\Gamma \times \Gamma)$ of all subsets of $\Gamma \times \Gamma$ is a compact, Hausdorff space (because it is naturally homeomorphic to the infinite Cartesian product $\{0,1\}^{\Gamma \times \Gamma}$ ). It is easy to see that the complement of $\mathcal{O}$ is open, so $\mathcal{O}$ is compact.

(2) The image of the basic open set $U_{\lambda_{1}, \lambda_{2}, \ldots, \lambda_{r}}$ under an element $\gamma$ of $\Gamma$ is the basic open set $U_{\lambda_{1} \gamma, \lambda_{2} \gamma, \ldots, \lambda_{r} \gamma}$.

\section{Amenability}

The following observation is immediate from Lemma 1.2 and the definition of amenability.

Lemma 2.1 Let $\Gamma$ be a left-orderable, amenable group. Then there is a $\Gamma$-invariant probability measure on the space $\mathcal{O}$ of left-invariant orders on $\Gamma$.

Remark 2.2 The above lemma is the only use that will be made of amenability. Hence, in the statements of Theorem A, Theorem B, and Corollary C, amenability can be replaced with the assumption that there is a $\Gamma$-invariant probability measure on $\mathcal{O}$.

\section{Poincaré Recurrence Theorem}

We recall the following classical result that can be found in almost any textbook on Ergodic Theory. For the convenience of the reader, we include a short proof.

Proposition 3.1 (Poincaré Recurrence Theorem [13, page 7]) Suppose

- $X$ is a measure space with probability measure $\mu$,

- $T: X \rightarrow X$ is an invertible, measurable map that preserves the measure $\mu$, and

- $A$ is any measurable subset of $X$.

Then there is a measurable subset $Z$ of $X$ with $\mu(Z)=0$, such that, for every $a \in A \backslash Z$, there is a sequence of positive integers $n_{i} \rightarrow \infty$, such that $T^{n_{i}}(a) \in A$ for every $i$. 
Proof For $n \in \mathbb{Z}^{+}$, let

$$
A_{n}=\bigcup_{k=1}^{\infty} T^{-k n}(A) .
$$

For each $a \in \bigcap_{n=1}^{\infty} A_{n}$, there is a sequence of positive integers $n_{i} \rightarrow \infty$, such that $T^{n_{i}}(a) \in A$ for every $i$. Thus, it suffices to show that $\mu\left(A \backslash A_{n}\right)=0$.

Suppose $\mu\left(A \backslash A_{n}\right)>0$. Since the sets

$$
T^{-n}\left(A \backslash A_{n}\right), T^{-2 n}\left(A \backslash A_{n}\right), T^{-3 n}\left(A \backslash A_{n}\right), \ldots
$$

all have the same measure (and $\mu(X)<\infty$ ), they cannot all be disjoint. Hence, there exist $k>\ell$, such that $T^{-k n}\left(A \backslash A_{n}\right) \cap T^{-\ell n}\left(A \backslash A_{n}\right) \neq \varnothing$. By applying $T^{\ell n}$, we may assume $\ell=0$. Therefore

$$
\varnothing \neq T^{-k n}\left(A \backslash A_{n}\right) \cap\left(A \backslash A_{n}\right) \subset T^{-k n}(A) \cap\left(A \backslash T^{-k n}(A)\right)=\varnothing .
$$

This is a contradiction.

Definition 3.2 A left-invariant order $\prec$ on a group $\Gamma$ is recurrent for every cyclic subgroup iff for every $\gamma \in \Gamma$ and every (finite) increasing sequence $\lambda_{1} \prec \lambda_{2} \prec \cdots \prec \lambda_{r}$ of elements of $\Gamma$, there exist positive integers $n_{i} \rightarrow \infty$, such that

$$
\lambda_{1} \gamma^{n_{i}} \prec \lambda_{2} \gamma^{n_{i}} \prec \cdots \prec \lambda_{r} \gamma^{n_{i}}
$$

for every $i$.

\section{Corollary 3.3 Suppose}

- $\Gamma$ is a left-orderable group that is countable, and

- there exists a $\Gamma$-invariant probability measure $\mu$ on the space $\mathcal{O}$ of left-invariant orders on $\Gamma$.

Then $\Gamma$ admits a left-invariant order that is recurrent for every cyclic subgroup.

Proof For each $\gamma \in \Gamma$ and each sequence $\lambda_{1}, \lambda_{2}, \ldots, \lambda_{r}$ of distinct elements of $\Gamma$, we may apply the Poincaré Recurrence Theorem with

- the space $\mathcal{O}$ in the role of $X$,

- the transformation $\gamma^{-1}$ in the role of $T$, and

- the basic open set $U_{\lambda_{1}, \ldots, \lambda_{r}}$ in the role of $A$.

We conclude that there is a set $Z_{\gamma, \lambda_{1}, \ldots, \lambda_{r}}$ of measure 0 in $\mathcal{O}$, such that: 
if $\prec$ is any left-invariant order on $\Gamma$, such that

- $\lambda_{1} \prec \lambda_{2} \prec \cdots \prec \lambda_{r}$, and

- $\prec$ is not in $Z_{\gamma, \lambda_{1}, \ldots, \lambda_{r}}$,

then there exist positive integers $n_{i} \rightarrow \infty$, such that

$$
\lambda_{1} \gamma^{n_{i}} \prec \lambda_{2} \gamma^{n_{i}} \prec \cdots \prec \lambda_{r} \gamma^{n_{i}} \quad \text { for every } i \text {. }
$$

The union of all of the sets $Z_{\gamma, \lambda_{1}, \ldots, \lambda_{r}}$ has measure zero (because it is a countable union of sets of measure 0 ), so there is a left-invariant order $\prec$ that does not belong to any $Z_{\gamma, \lambda_{1}, \ldots, \lambda_{r}}$. This order is recurrent for every cyclic subgroup.

Combining the above corollary with Lemma 2.1 immediately yields the following conclusion.

Corollary 3.4 If $\Gamma$ is any countable, left-orderable, amenable group, then $\Gamma$ admits a left-invariant order that is recurrent for every cyclic subgroup.

\section{Recurrent orders and indicable groups}

The main result of this section is Corollary 4.4. It is an almost immediate consequence of a known result (Theorem 4.2 below), but, for the convenience of the reader, we provide a short proof that is fairly self contained.

Definition 4.1 $[4$, Lemma 6.6.2(1,3), page 121] A left-invariant order $\prec$ on a group $\Gamma$ is Conradian iff for every $\gamma, \lambda \in \Gamma$, such that $\gamma \succ e$ and $\lambda \succ e$, there exists $n \in \mathbb{Z}^{+}$, such that $\lambda \gamma^{n} \succ \gamma$.

Theorem 4.2 [4, Theorem 6.J, page 128] A group is locally indicable if and only if it admits a Conradian left-invariant order.

Lemma 4.3 If a left-invariant order is recurrent for every cyclic subgroup, then the order is Conradian.

Proof If $\lambda \succ e$, then recurrence implies there exists $n \in \mathbb{Z}^{+}$, such that $\lambda \gamma^{n} \succ e \gamma^{n}=\gamma^{n}$. If, in addition, we have $\gamma \succ e$, then $\gamma^{n} \succeq \gamma$, so transitivity implies $\lambda \gamma^{n} \succ \gamma$.

Combining Lemma 4.3 with Theorem 4.2 yields the following result:

Corollary 4.4 If a group admits a left-invariant order that is recurrent for every cyclic subgroup, then the group is locally indicable.

Algebraic 83 Geometric Topology, Volume 6 (2006) 
Before providing a proof of Corollary 4.4 that does not rely on Theorem 4.2, let us recall some elementary properties of left-ordered groups.

Remark 4.5 Let $\prec$ be a left-invariant order on a group $\Gamma$.

(1) To say that a subgroup $\Lambda$ of $\Gamma$ is convex means that if $\lambda_{1} \prec \gamma \prec \lambda_{2}$, with $\lambda_{1}, \lambda_{2} \in \Lambda$, then $\gamma \in \Lambda$ [4, page 31]. (Because $\prec$ is left invariant, it suffices to verify this condition in the special case where $\lambda_{1}=e$.)

(2) If $\Lambda$ is a convex, proper subgroup of $\Gamma$, then:

(a) $\Lambda$ is an interval in the total order $(\Gamma, \prec)$ (so left invariance implies that each left coset of $\Lambda$ is also an interval), so

(b) $\prec$ induces a well-defined total order on the space of left cosets of $\Lambda$, so

(c) $\Lambda$ is bounded above (by any positive element of $\Gamma$ that does not belong to $\Lambda$ ) and bounded below.

(3) If $\Gamma$ is finitely generated, then Zorn's Lemma implies that $\Gamma$ has a maximal (proper) convex subgroup. The convex subgroups of $\Gamma$ are totally ordered by inclusion [4, Lemma 3.1.2, page 32], so this maximal convex subgroup is unique.

(4) The order $\prec$ is Archimedean if, for all nontrivial $\gamma, \lambda \in \Gamma$, there exists $n \in \mathbb{Z}$, such that $\gamma \preceq \lambda^{n}$ [4, page 55]. It is well known (and not difficult to show [4, Corollary 4.1.3, page 56]) that any group admitting an Archimedean left-invariant order must be abelian (and torsion free).

Direct proof of Corollary 4.4 Assume that $\prec$ is a left-invariant order on a finitely generated group $\Gamma$, and that $\prec$ is recurrent for every cyclic subgroup.

Begin by noting, for $\lambda_{1}, \lambda_{2}, \ldots, \lambda_{r}, \lambda \in \Gamma$, that if $\max \left\{\lambda_{1}^{ \pm 1}, \ldots, \lambda_{r}^{ \pm 1}\right\} \preceq \lambda$, then there exists $n \in \mathbb{Z}^{+}$, such that

$$
\lambda_{1} \lambda_{2} \cdots \lambda_{r} \preceq \lambda^{n}
$$

To see this, choose (by induction on $r$ ) some $m \in \mathbb{Z}^{+}$, such that $\lambda_{2} \lambda_{3} \cdots \lambda_{r} \preceq \lambda^{m}$, and then choose (by recurrence of $\prec$ ) some $n \in \mathbb{Z}^{+}$, such that

$$
\lambda_{1} \lambda^{m n} \preceq \lambda^{m} \lambda^{m n}=\lambda^{m(n+1)} .
$$

Then we have $\lambda_{2} \lambda_{3} \cdots \lambda_{r} \preceq \lambda^{m} \preceq \lambda^{m n}$, so

$$
\lambda_{1} \lambda_{2} \lambda_{3} \cdots \lambda_{r} \preceq \lambda_{1} \lambda^{m n} \preceq \lambda^{m(n+1)},
$$

as desired.

Since $\Gamma$ is finitely generated, it has a maximal (proper) convex subgroup $\Lambda$, which is unique. For any $\gamma \in \Gamma^{+}$, the set

$$
\Lambda_{\gamma}=\left\{\lambda \in \Gamma \mid \lambda^{n} \prec \gamma \text { for all } n \in \mathbb{Z}\right\}
$$

Algebraic $\mathcal{E} \mathcal{G}$ Geometric Topology, Volume 6 (2006) 
is obviously closed under inverses, and the observation of the preceding paragraph implies that it is closed under multiplication and that it is convex; hence, it follows from the uniqueness of the maximal convex subgroup that $\Lambda_{\gamma} \subset \Lambda$. Thus,

(*) $\quad \Lambda$ contains every subgroup of $\Gamma$ that is bounded above.

The reversal of a recurrent order is recurrent, so the same argument implies

$(* *) \quad \Lambda$ contains every subgroup of $\Gamma$ that is bounded below.

Since $\Lambda$ is a convex proper subgroup, it is bounded both above (by some $\lambda_{+}$) and below (by some $\lambda_{-}$). For $\gamma \in \Gamma$, and any $\lambda \in \Lambda$, we have

$$
\gamma \succ e \Longrightarrow \gamma^{-1} \prec e \Longrightarrow \lambda \gamma^{-1} \prec \lambda \preceq \lambda_{+} \Longrightarrow \gamma \lambda \gamma^{-1} \prec \gamma \lambda_{+} .
$$

Similarly,

$$
\gamma \prec e \Longrightarrow \gamma \lambda \gamma^{-1} \succ \gamma \lambda_{-} .
$$

Thus, for any $\gamma \in \Gamma$, the conjugate $\gamma \Lambda \gamma^{-1}$ is either bounded above (by $\gamma \lambda_{+}$) or bounded below (by $\gamma \lambda_{-}$). From (*) and (**), we conclude that $\gamma \Lambda \gamma^{-1} \subset \Lambda$. Therefore, $\Lambda$ is normal in $\Gamma$.

The order induced on the quotient group $\Gamma / \Lambda$ is Archimedean (because (*) implies no nontrivial subgroup of $\Gamma / \Lambda$ is bounded above), so $\Gamma / \Lambda$ must be abelian and torsion free. Then the structure of finitely generated abelian groups implies that $\Gamma / \Lambda$ has an infinite cyclic quotient.

The converse of Corollary 4.4 is false:

Example 4.6 Let $F$ be a free subgroup of finite index in $\operatorname{SL}(2, \mathbb{Z})$, and let $\Gamma$ be the natural semidirect product $F \ltimes \mathbb{Z}^{2}$. Then $\Gamma$ is locally indicable, but has no left-invariant order that is recurrent for every cyclic subgroup.

Proof Since free groups and $\mathbb{Z}^{2}$ are locally indicable, it is clear that $\Gamma$ is locally indicable.

Suppose $\prec$ is a left-invariant order on $\Gamma$ that is recurrent for every cyclic subgroup. (This will lead to a contradiction.) For a matrix $T$ in $F$ and a vector $v$ in $\mathbb{Z}^{2}$, let us use $\bar{T}$ and $\bar{v}$ to represent the corresponding elements of $F \ltimes \mathbb{Z}^{2}$, so $\bar{T} \bar{v} \bar{T}^{-1}=\overline{T(v)}$.

There is a nonzero linear functional $f: \mathbb{R}^{2} \rightarrow \mathbb{R}$, such that $f(v)>0 \Rightarrow \bar{v} \succ e$, for all $v \in \mathbb{Z}^{2}$. Let $T$ be a hyperbolic matrix in $F$, with eigenvalues $\alpha_{1}, \alpha_{2}>0$ and corresponding eigenvectors $v_{1}$ and $v_{2}$. Since $v_{1}$ and $v_{2}$ are linearly independent, we may assume $f\left(v_{1}\right) \neq 0$. Furthermore, we may assume $f\left(v_{1}\right)>0$ and $\alpha_{1}>1$ (so 
$\alpha_{2}<1$ ), by replacing $v_{1}$ with $-v_{1}$ and/or $T$ with $T^{-1}$, if necessary. Let $g: \mathbb{R}^{2} \rightarrow \mathbb{R}$ be the (unique) linear functional that satisfies $g\left(v_{1}\right)=1$ and $g\left(v_{2}\right)=0$.

Given any $v \in \mathbb{Z}^{2}$, such that $f(v)>0$, recurrence provides a sequence $n_{i} \rightarrow \infty$, such that $\bar{v} \bar{T}^{-n_{i}} \succ \bar{T}^{-n_{i}}$ for every $i$. By left invariance, this implies ${\overline{T^{n_{i}}(v)}}=\bar{T}^{n_{i}} \bar{v} \bar{T}^{-n_{i}} \succ$ $e$, so $f\left(T^{n_{i}}(v)\right) \geq 0$. Since

$$
\lim _{n \rightarrow \infty} \frac{1}{\alpha_{1}^{n}} f\left(T^{n}(v)\right)=f\left(\lim _{n \rightarrow \infty} \frac{1}{\alpha_{1}^{n}} T^{n}(v)\right)=f\left(g(v) v_{1}\right)=g(v) f\left(v_{1}\right),
$$

we conclude that $g(v) \geq 0$.

Since $v$ is an arbitrary element of $\mathbb{Z}^{2}$ with $f(v)>0$, this implies that ker $f=\operatorname{ker} g$ is an eigenspace of $T$. But $T$ is an arbitrary hyperbolic matrix in $F$, and it is easy to show that there are two (conjugate) hyperbolic matrices in $F$ that do not have a common eigenspace. This is a contradiction.

\section{References}

[1] R G Burns, V W D Hale, A note on group rings of certain torsion-free groups, Canad. Math. Bull. 15 (1972) 441-445 MR0310046

[2] I M Chiswell, P H Kropholler, Soluble right orderable groups are locally indicable, Canad. Math. Bull. 36 (1993) 22-29 MR1205890

[3] É Ghys, Groups acting on the circle, Enseign. Math. (2) 47 (2001) 329-407 MR1876932

[4] A M W Glass, Partially ordered groups, Series in Algebra 7, World Scientific, River Edge, NJ (1999) MR1791008

[5] P H Kropholler, Amenability and right orderable groups, Bull. London Math. Soc. 25 (1993) 347-352 MR1222727

[6] P A Linnell, Left ordered amenable and locally indicable groups, J. London Math. Soc. (2) 60 (1999) 133-142 MR1721820

[7] P A Linnell, Left ordered groups with no non-abelian free subgroups, J. Group Theory 4 (2001) 153-168 MR1812322

[8] P Longobardi, M Maj, A H Rhemtulla, Groups with no free subsemigroups, Trans. Amer. Math. Soc. 347 (1995) 1419-1427 MR1277124

[9] P Longobardi, M Maj, A H Rhemtulla, When is a right orderable group locally indicable?, Proc. Amer. Math. Soc. 128 (2000) 637-641 MR1694872

[10] J-P Pier, Amenable locally compact groups, Pure and Applied Mathematics, A WileyInterscience Publication, John Wiley \& Sons, New York (1984) MR767264 
[11] A H Rhemtulla, Polycyclic right-ordered groups, from: "Algebra, Carbondale 1980 (Proc. Conf., Southern Illinois Univ., Carbondale, Ill., 1980)", Lecture Notes in Math. 848, Springer, Berlin (1981) 230-234 MR613189

[12] A S Sikora, Topology on the spaces of orderings of groups, Bull. London Math. Soc. 36 (2004) 519-526 MR2069015

[13] Y G Sinai, Introduction to ergodic theory, Translated by V. Scheffer, Mathematical Notes 18, Princeton University Press (1976) MR0584788

Department of Mathematics and Computer Science, University of Lethbridge Lethbridge, Alberta, T1K 3M4, Canada

Dave.Morris@uleth.ca

http://people.uleth.ca/ dave.morris/

Received: 9 June 2006 